\title{
Developing a Simulated Environment to Study Naturalistic Decision Making Processes
}

\author{
Dorian Soru ${ }^{1}$, Paolo Cottone ${ }^{1}$, Gianmarco Alto $\grave{2}^{2}$, Valentina Schiavinato ${ }^{1}$ and \\ Giuseppe Mantovani ${ }^{1}$ \\ ${ }^{1}$ Department of General Psychology \\ ${ }^{2}$ Department of Developmental and Socialization Psychology \\ University of Padua, Italy \\ \{dorian.soru / paolo.cottone / gianmarco.altoe / valentina.schiavinato / giuseppe.mantovani\}@unipd.it
}

\begin{abstract}
Motivation - Combination of qualitative and quantitative methodologies to develop a simulated environment to study decision-making processes from a NDM perspective. Research approach Discourse analysis to find interpretative repertoires, use of Visual Analog Scales and ANOVA to validate the repertoires. Findings/Design - Usefulness of qualitative and quantitative methodologies to develop the simulation, importance of a rigorous validation. Research limitations/Implications - Need for comparison with a real website in further studies. Originality/Value - Use of NDM perspective to investigate processes that were studied just from a DM perspective. Take away message - It is possible to study decision-making by naturally simulating them with computer technology in a laboratory.
\end{abstract}

\section{Keywords}

Simulation, validation, house-renting decisions, ecological validity.

The present research focuses on the definition of a procedure that can be used to develop a simulation to study decisionmaking processes in everyday life situations. More specifically, the simulated environment developed within this study should enable researchers to find, in further studies, the role of gender in decision-making processes. Despite the fact that gender is a topic investigated in many studies - in fact a large number of papers covering different contexts can be easily found, from professional and organisational to economic fields - it is also one of the most vexing; and the economic field appears to be the one which shows the most different and conflicting findings (Sproles \& Kendall 1986, Patel 2008). One of the limitations of many studies in economical decision-making is that they use a research paradigm which is unable to manage those situational issues embodied in decision making processes. This research deals with this limitation by developing a simulated environment that enables researchers to follow Naturalistic Decision Making (NDM) (Lipshitz et al. 2001) guidelines: this paradigm provides a framework for researchers to study decision making in a simulated environment (Elliott et al 2007), focusing more on processes than on the result of decision making.

The present research involves three different steps: a preliminary study, aimed to investigate the first analysis of the situation based on qualitative methods, followed by two additional studies aimed to validate the simulated environment. All three studies take place in a mid-sized northern Italian town. This study is also an attempt to document, with a NDM approach, a task - house-renting decision-making - that typically does not fit completely with the NDM guidelines specified by Lipshitz et al. (2007). This type of activity is usually studied from non-naturalistic perspectives. Decisions about house-renting usually do not seem to be critical, stressful and high-tasked activities, yet this situation could fit all these NDM characteristics, depending on the participants to the study. The town where this research takes place hosts a very prestigious university and, for this reason, university students are of great importance to the town's economy. Many students who leave high school and enter the university do not know the town's geography. Moreover, they can often look for a house only when they pass an entry test given by the faculty they choose. For many students it quickly becomes critical to find a house before they start their classes (so it becomes an high-tasked activity) in a town they do not know (so it is a stressful task).

The present study investigates house-renting decisions in a simulated web site. Participants in the preliminary study $(\mathrm{N}=14 ; \mathrm{F}=7 \mathrm{M}=7$; Mean Age = 22.53) were told that the university would give them a scholarship to rent a house, and that they should choose from among five houses on the website. Each participant was asked to navigate through a website similar (for logo and colours) to that of the university, where s/he could choose among the houses. Video and audio recorded sessions and interviews, amounting to 200 minutes of footage, were transcribed and analysed through discourse analysis, in order to discover the interpretative repertoires (Edley, 2001) used by participants for their choice. We discovered two repertoires that were used to choose: one called "Comfortableness" and another called "Pleasantness". First and second validation studies validated, using a quantitative analysis, the descriptions and pictures of the houses presented in the website and also the data collection system embedded in the website.

Participants to the first validation study $(\mathrm{N}=66 ; 20 \mathrm{M}, 46 \mathrm{~F} . \mathrm{N}=63)$ were asked to navigate the website and judge the 
descriptions and the pictures of the house on two axes (comfortable/uncomfortable for the "Comfortableness" dimension and pleasant/unpleasant for the "Pleasantness" dimension). We decided to measure these categorizations by using a continuum (a Visual Analog Scale) instead of a Likert scale in order to get an interval measure, instead of the quasi-interval data obtained by using a Likert scale. Each categorization was divided by the line length, such that we obtained a scalar variable ranging between 0.00 and 1.00 (where 0.00 corresponds to the minimum judgement for comfortableness/pleasantness and 1.00 corresponds to the maximum for both adjectives). All participants categorized all houses. Following the experimental design described above, we performed a $6 \times 2$ repeated measures ANOVA with JUDGEMENT as dependent variable and HOUSE (1/2/3/4/5/6) and ADJECTIVE (comfortableness vs. pleasantness) as within factors. The sphericity assumption was met for house: $\chi 2(14)=20.656, p=0.111$ and for the interaction: $\chi 2(14)=18.154, p=0.200$. The main effect of HOUSE was significant: $F(5,325)=31.851, p<0.001$. Houses 1,3 and 4 had the highest judgements for comfortableness and for pleasantness as well, houses 5 and 6 had a lower judgement and house 2 had the lowest judgement. The main effect of ADJECTIVE was not significant: $F(1,65)=0.007, p=0.933$ : Overall, there is not a difference between these two judgements. The interaction was significant: $F(5,325)=3.270$, $\mathrm{p}<0.05$. To explore the interaction, six one sample t-tests, comparing to zero the difference between comfortable and pleasantness, were performed using the false discovery rate (FDR) correction (Benjamini \& Hochberg, 1995). No significant differences were found. Even if there is a global effect of the interaction, if we consider each house distinctly, difference is never statistically significant.

The second validation study was very similar to the previous one. The only difference was that we tested the influence of house presentation order: each participant $(\mathrm{N}=63, \mathrm{~F}=41, \mathrm{M}=22$, Mean Age=22.90) was randomly assigned to one of the three presentation orders we defined based on our expectation. We performed a $6 \times 2 \times 3$ repeated measures ANOVA with JUDGEMENT as dependent variable and HOUSE (1/2/3/4/5/6) and ADJECTIVE (comfortableness vs pleasantness) as within factors and ORDER (1/2/3) as between factor. The sphericity assumption was met for HOUSE: $\chi 2(14)=13.211, p=0.510$ but was not met for the interaction: $\chi 2(14)=39.005, p<0.001$. Greenhouse-Geisser correction will be used for the interaction. Results show that there was no significant main effect or interaction for ORDER so we performed a 6x3 repeated measure analysis deleting ORDER. JUDGEMENT was the dependent variable, HOUSE $(1 / 2 / 3 / 4 / 5 / 6)$ and ADJECTIVE (comfortableness vs pleasantness) were within factors. The sphericity assumption was met for house: $\chi 2(14)=12.590, \mathrm{p}=0.559$ but was not met for the interaction: $\chi 2(14)=39.602$, $p<0.001$. GreenhouseGeisser correction will be used for the interaction. The main effect of HOUSE was significant: $F(5,310)=9.314$, $\mathrm{p}<0.001$. House $5 \mathrm{had}$ a high judgements for comfortableness and for pleasantness as well; houses 2 and 3 had a higher judgement for pleasantness than for comfortableness; houses 1, 4 and 6 had a higher judgement for comfortableness than for pleasantness. The main effect of ADJECTIVE was not significant: $F(1,62)=0.764, p=0.385$ : Overall, there is not a difference between these two judgements. The interaction was significant: $F(4.092,253.697)=9.374, p<0.001$. To explore the interaction, six one-sample t-tests, comparing to zero the difference between comfortable and pleasantness, were performed, using the FDR correction. Difference for house 1 was not significantly different from $0: t(61)=1.612$, $\mathrm{p}=0.168$; difference for house 2 was significantly different from $0: \mathrm{t}(62)=-4.067, \mathrm{p}<0.001$; difference for house 3 was not significantly different from 0 : $\mathrm{t}(62)=-0.517, \mathrm{p}=0.729$; difference for house 4 was significantly different from 0 : $\mathrm{t}(62)=3.300, \mathrm{p}<0.01$; difference for house 5 was not significantly different from $0: \mathrm{t}(62)=0.054$, $\mathrm{p}=0.957$; difference for house 6 was significantly different from $0: \mathrm{t}(62)=2.589, \mathrm{p}<0.05$.

The analysis shows that houses 1, 3 and 5 were judged neutral (comfortable and pleasant at the same time), houses 4 and 6 were judged more comfortable than pleasant and house 2 was judged more pleasant than comfortable. Analyses have shown that qualitative and quantitative methods can be joined to construct a simulated environment for NDM research. Results of our preliminary study were confirmed in validation studies: A main effect of HOUSE and of HOUSE $x$ ADJECTIVE was found both in the first and in the second validation study. However, no significant difference was found in multiple t-test comparisons for the first validation study: this suggests the importance of the rigorous validation of experimental environments that was performed on the second validation study. Further studies need to be performed in order to better compare the simulated environment with a real house-renting company website.

\section{REFERENCES}

Benjamini, Y., and Hochberg, Y. (1995). Controlling the false discovery rate: a practical and powerful approach to multiple testing. Journal of the Royal Statistical Society, Series B, 57, 289-300.

Edley, N. (2001) Analysing Masculinity: Interpretative Repertoires, Subject Positions and Ideological Dilemmas in M. Wetherell, S. Taylor and S. J.Yates (Eds.) Discourse as Data: A guide for Analysis (pp. 189-228). London: Sage.

Elliott, T., Welsh, M., Nettelbeck, T., \& Mills, V. (2007). Investigating Naturalistic Decision Making in a simulated microworld: what questions should we ask? Behaviour Research Methods, 39(4), 901-910.

Lipshitz, R., Klein, G. A., Orasanu, J. \& Salas, E. (2001). Focus article: Taking Stock of Naturalistic Decision Making. Journal of Behavioral Decision Making, 14,331-352.

Patel, V. (2008). Consumer Decision Making Styles in Shopping Malls: An Empirical Study. In U. Dhar, V. V. Nath, S. K. Nair, \& P. K. Yadav (Eds.) New Age Marketing: Emerging Realities (pp. 627-637). New Delhi: Excel Books.

Sproles, G. B., \& Kendall, E. L. (1986). A methodology for profiling consumers' decision-making styles. Journal of Consumer Affairs, 20(2), 267-279. 